\title{
Impact of branched-chain amino acids and frailty on the management of lenvatinib-related fatigue in patients with hepatocellular carcinoma
}

\author{
Shigeo Shimose', Shunji Koya ${ }^{2}$, Takumi Kawaguchi', Keisuke Hirota², Sachiyo Yoshio ${ }^{4}$, Takashi Niizeki ${ }^{1}$, \\ Hiroo Matsuse ${ }^{2,3}$, and Takuji Torimura ${ }^{1}$ \\ 'Division of Gastroenterology, Department of Medicine, Kurume University School of Medicine, Kurume; ${ }^{2}$ Division of Rehabilitation, \\ Kurume University Hospital, Kurume; ${ }^{3}$ Department of Orthopedics, School of Medicine, Kurume University, Kurume; ${ }^{4}$ Department of \\ Liver Disease, Research Center for Hepatitis and Immunology, National Center for Global Health and Medicine, Kohnodai, Japan
}

Keywords: Lenvatinib; Fatigue; Amino acids, Branched-chain; Frailty; Carcinoma, Hepatocellular

Dear Editor,

Molecular target agents (MTAs) are an important treatment options for patients with unresectable hepatocellular carcinoma (HCC). ' Lenvatinib (LEN), an oral multi-kinase inhibitor, has a higher response rate compared to other MTAs, including sorafenib. ${ }^{2}$ However, fatigue is one of the adverse events (AEs) of LEN, limiting its clinical application. ${ }^{3}$

Fatigue is underinvestigated and often undertreated in patients with HCC. However, frailty reportedly outperforms other factors in predicting fatigue in patients with breast cancer undergoing radiotherapy. ${ }^{4}$ Additionally, malnutrition can cause fatigue in patients with chronic liver disease. ${ }^{5}$ The depletion of serum branched-chain amino acid (BCAA) levels was associated with fatigue in patients on hemodialysis. ${ }^{6}$ Thus, frailty and BCAA are possible factors associated with LEN-related fatigue. Herein, we performed a data-mining analysis based on artificial intelligence and found that BCAA supplementation and frailty were associat- ed with LEN-related fatigue in patients with unresectable HCC.

We enrolled 42 consecutive patients with HCC treated using LEN in this observational study approved by the Institutional Review Board of Kurume University (approval number: 19098). All patients were regularly followed until death or study cessation (March 31, 2021). LEN (Eisai Co., Ltd, Tokyo, Japan) was orally administered at a dose of $12 \mathrm{mg} /$ day in patients with body weight $\geq 60 \mathrm{~kg}$ or $8 \mathrm{mg} /$ day in patients with body weight $<60 \mathrm{~kg}$, and it was discontinued when any AEs of unacceptable grade 2 or $\geq$ grade 3 occurred.

Any grade fatigue occurred in 30.9\% (13/42) of enrolled patients, and grade $\geq 2$ fatigue occurred in $26.2 \%$ (11/42) of enrolled patients. There were no significant differences in age, sex, or body mass index between the grade $<2$ and grade $\geq 2$ fatigue groups. No significant differences were observed in the albuminbilirubin (ALBI) grade or Barcelona Clinic Liver Cancer (BCLC) stage between the two groups. In patients with grade $\geq 2$ fatigue, $9.1 \%(1 / 11)$ were treated with BCAA supplementation. In con-

\section{Abbreviations:}

AEs, adverse events; ALBI, albumin-bilirubin; BCAA, branched-chain amino acid; BCLC, Barcelona Clinic Liver Cancer; HCC, hepatocellular carcinoma; LEN, lenvatinib; MTAs, molecular target agents

\section{Corresponding author : Takumi Kawaguchi}

Division of Gastroenterology, Department of Medicine, Kurume University School of Medicine, 67 Asahi-machi, Kurume 830-0011, Japan

Tel: +81-942-31-7627, Fax: +81-942-31-2623

E-mail: takumi@med.kurume-u.ac.jp

https://orcid.org/0000-0002-7064-4325 
trast, $38.7 \%(12 / 31)$ of patients with grade $<2$ fatigue were treated with BCAA supplementation.

Independent factors associated with grade $\geq 2$ fatigue were examined by logistic regression analysis with a stepwise procedure using the following 14 variables: age, sex, body mass index, performance status, BCLC stage, vascular invasion of HCC, extrahepatic spread, initial dose of LEN, period of LEN treatment, ALBI grade, diabetes mellitus, sarcopenia, frailty, and BCAA supplementation. In a stepwise procedure, only BCAA supplementation was selected as an exploratory variable for logistic regression analysis. Logistic regression analysis identified BCAA supplementation as an independent factor associated with grade $\geq 2$ fatigue (odds ratio, $0.158 ; 95 \%$ confidence interval, $0.008-0.089 ; P=0.049$ ). The reason why BCAA was associated with a low incidence of fatigue remains unclear. However, the following are possible explanations: first, fatigue can be caused by increased serotonin synthesis in the brain, which is derived from tryptophan. ${ }^{7.8}$ BCAA suppresses the transport of tryptophan across the blood-brain barrier and reduces brain serotonin. ${ }^{9}$ Second, muscle plays a major role in the catabolic reaction of kynurenine, which causes fatigue. ${ }^{10}$ LEN induces muscle atrophy, ${ }^{11}$ leading to an increase in serum kynurenine levels. ${ }^{12}$ On the other hand, BCAA stimulates muscle protein synthesis and suppresses muscle atrophy. ${ }^{13}$ Thus, BCAA supplementation may contribute to suppressing LEN-related fatigue through alteration of tryptophan and kynurenine metabolism.

A decision-tree analysis then identified BCAA supplementation as the most important classifier for grade $\geq 2$ fatigue. In patients with BCAA supplementation, $7.7 \%$ (1/13) showed grade $\geq 2$ fatigue. In contrast, $34.5 \%$ (10/29) showed grade $\geq 2$ fatigue in patients without BCAA supplementation (Fig. 1). In patients without

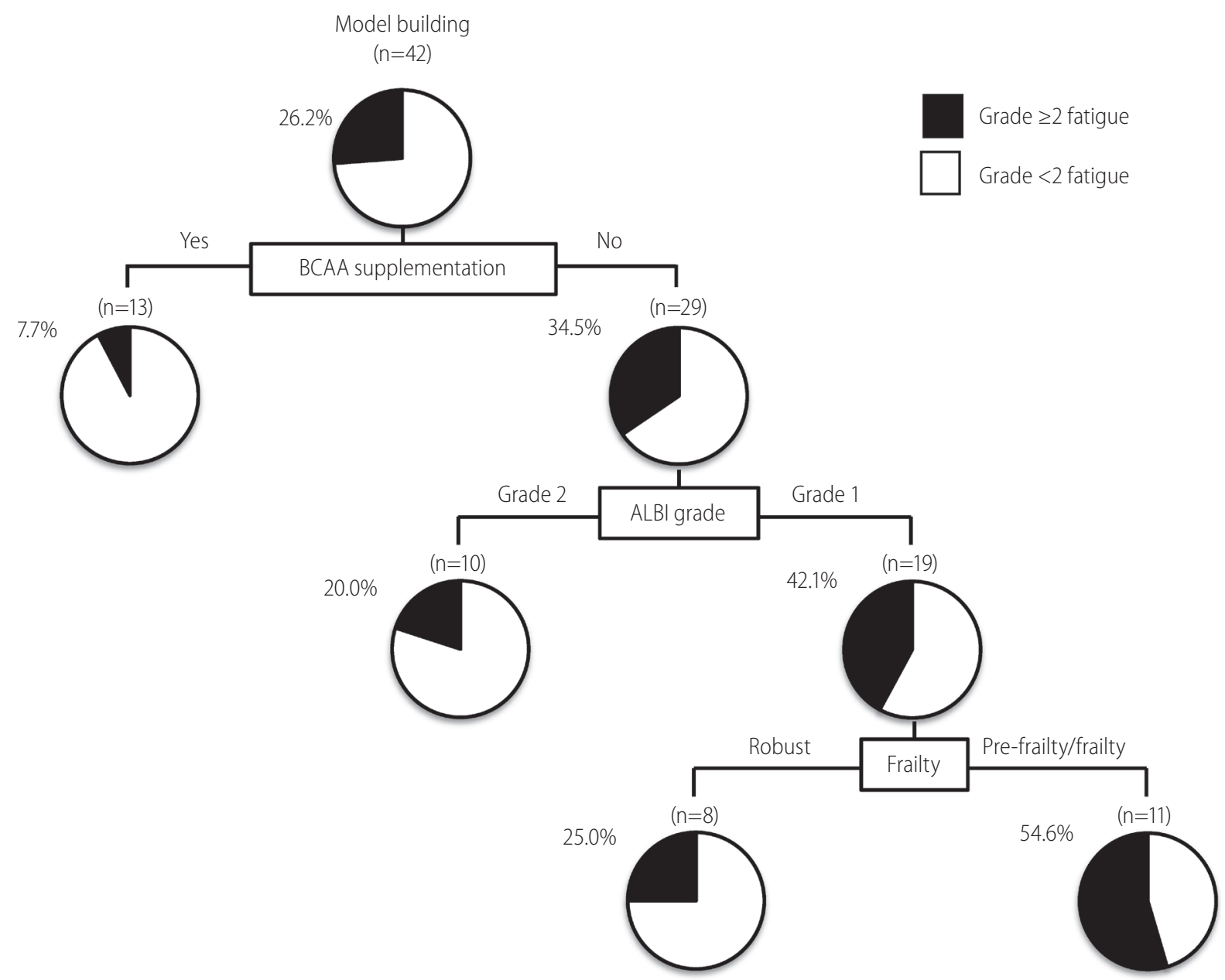

Figure 1. Decision-tree algorithm for grade $\geq 2$ fatigue during lenvatinib treatment in patients with unresectable hepatocellular carcinoma. The pie graphs indicate the percentage of grade $<2$ fatigue (white)/grade $\geq 2$ fatigue (black) in each group. BCAA, branched-chain amino acid; ALBl, albuminbilirubin. 
BCAA supplementation, the second and third classifiers were ALBI grade and frailty, respectively. In patients with ALBI grade 1 and pre-frailty/frailty, $54.6 \%$ (6/11) showed grade $\geq 2$ fatigue (Fig. 1). Thus, frailty may be associated with LEN-related fatigue. Uojima et al. ${ }^{14}$ reported that decreased skeletal muscle mass was associated with the onset of severe AEs. Moreover, Endo et al..$^{15}$ reported that muscle strength was associated with the onset of severe AE in patients with HCC treated with LEN. Thus, these findings suggest that frailty is a risk factor for grade $\geq 2$ fatigue, and frailty should be evaluated before LEN treatment even in patients with HCC with ALBI grade 1.

This study had some limitations. First, this was a single-center study, and the selection bias of the enrolled patients may have affected our results. Second, the total sample size and event number were small; therefore, we employed a stepwise procedure to select exploratory variables for logistic regression analysis. Third, we could not evaluate the interventional effects of BCAA supplementation and exercise therapy on fatigue. Further study should be designed as a large-scale multicenter study focusing on the effect of BCAA supplementation and cancer rehabilitation on fatigue in patients with unresectable HCC treated with LEN.

In conclusion, grade $\geq 2$ fatigue occurred in approximately $25 \%$ of patients with unresectable HCC treated with LEN. BCAA and frailty were associated with LEN-related fatigue. Thus, BCAA supplementation and maintenance of physical function may be beneficial for the management of fatigue in patients with unresectable HCC treated with LEN.

\section{Authors' contributions}

All authors were responsible for the interpretation of data, drafting, and critical revision of the manuscript for important intellectual content.

\section{Acknowledgements}

This work was supported by the Japan Society for the Promotion of Science (JSPS) Grant-in-Aid for Scientific Research (C) JP20K08395 and by the Research Program on Hepatitis from the Japan Agency for Medical Research and Development, AMED under $21 \mathrm{fk} 0210094$.

\section{Conflicts of Interest}

Takumi Kawaguchi received lecture fees from Janssen Pharmaceutical K.K., Mitsubishi Tanabe Pharma Corporation, and Otsuka Pharmaceutical Co., Ltd. The other authors declare no conflicts of interest.

\section{REFERENCES}

1. Ogasawara S, Ooka Y, Koroki K, Maruta S, Kanzaki H, Kanayama $K$, et al. Switching to systemic therapy after locoregional treatment failure: definition and best timing. Clin Mol Hepatol 2020;26:155162.

2. Kudo M. Recent Advances in Systemic Therapy for Hepatocellular Carcinoma in an Aging society: 2020 update. Liver Cancer 2020;9:640-662.

3. Shimose S, Iwamoto H, Niizeki T, Shirono T, Noda Y, Kamachi N, et al. Clinical significance of adverse events for patients with unresectable hepatocellular carcinoma treated with lenvatinib: a multicenter retrospective study. Cancers (Basel) 2020;12:1867.

4. Denkinger MD, Hasch M, Gerstmayer A, Kreienberg R, Nikolaus T, Hancke $K$. Predicting fatigue in older breast cancer patients receiving radiotherapy. A head-to-head comparison of established assessments. Z Gerontol Geriatr 2015;48:128-134.

5. Jones EA. Fatigue associated with chronic liver disease: a riddle wrapped in a mystery inside an enigma. Hepatology 1995;22:16061608.

6. Debnath S, Lorenzo C, Bansal S, Morales J, Rueda RO, Kasinath BS, et al. Branched-chain amino acids depletion during hemodialysis is associated with fatigue. Am J Nephrol 2020;51:565-571.

7. Lanser L, Kink P, Egger EM, Willenbacher W, Fuchs D, Weiss $G$, et al. Inflammation-induced tryptophan breakdown is related with anemia, fatigue, and depression in cancer. Front Immunol 2020;11:249.

8. Fosså A, Smeland KH, Fluge $\varnothing$, Tronstad KJ, Loge JH, Midttun $\varnothing$, et al. Metabolic analysis of amino acids and vitamin $\mathrm{B} 6$ pathways in lymphoma survivors with cancer related chronic fatigue. PLoS One 2020;15:e0227384.

9. Blomstrand E. A role for branched-chain amino acids in reducing central fatigue. J Nutr 2006;136:544S-547S.

10. Agudelo LZ, Femenía T, Orhan F, Porsmyr-Palmertz M, Goiny M, Martinez-Redondo $V$, et al. Skeletal muscle PGC-1a1 modulates kynurenine metabolism and mediates resilience to stress-induced depression. Cell 2014;159:33-45.

11. Uchikawa S, Kawaoka T, Namba M, Kodama K, Ohya K, Morio K, et al. Skeletal muscle loss during tyrosine kinase inhibitor treatment for advanced hepatocellular carcinoma patients. Liver Cancer 2020;9:148-155.

12. Kaiser $H, Y u$ K, Pandya C, Mendhe B, Isales CM, McGee-Lawrence $M E$, et al. Kynurenine, a tryptophan metabolite that increases with age, induces muscle atrophy and lipid peroxidation. Oxid Med Cell Longev 2019;2019:9894238.

13. Kawaguchi T, Izumi N, Charlton MR, Sata M. Branched-chain amino acids as pharmacological nutrients in chronic liver disease. Hepatology 2011;54:1063-1070.

14. Uojima H, Chuma M, Tanaka Y, Hidaka H, Nakazawa T, Iwabuchi 
Shigeo Shimose, et al.

Management of lenvatinib-related fatigue

$S$, et al. Skeletal muscle mass influences tolerability and prognosis in hepatocellular carcinoma patients treated with lenvatinib. Liver Cancer 2020;9:193-206.
15. Endo K, Kuroda H, Kanazawa J, Sato T, Fujiwara Y, Abe T, et al. Impact of grip strength in patients with unresectable hepatocellular carcinoma treated with lenvatinib. Cancers (Basel) 2020;12:2146. 